\title{
ANÁLISIS CUALITATIVO DE LAS RESPUESTAS DE LOS ALUMNOS SOBRE ASPECTOS DEL CURSO DEL PROCESO DE SOFTWARE PERSONAL
}

\author{
Juan Ángel Contreras ${ }^{1}$, Juan Arias $^{2}$, Violeta Hidalgo ${ }^{3}$ y Rafael Martín ${ }^{4}$ \\ 1,2y ^4niversidad de Extremadura, Centro Universitario de Mérida, España. 1jaconvas@unex.es; ;2 jarias@unex.es; \\ ${ }^{4}$ rmmartin@unex.es; \\ ${ }^{3}$ Universidad de Extremadura y Centro Universitario de Mérida, España. vhidalgo@unex.es
}

\begin{abstract}
Resumen. En este artículo se realiza el análisis mixto de las respuestas dadas por los alumnos sobre la impartición de un curso realizado en la asignatura de Ingeniería de Software y titulado el Proceso de Software Personal (PSP). Estas respuestas, para su análisis posterior, se han dividido en cuatro grupos atendiendo a cada una de las preguntas realizadas, y para cada una de ellas se han obtenido las categorías de análisis. Estas categorías de análisis nos han permitido agrupar las diferentes respuestas que han dado los alumnos a un cuestionario que posteriormente mostraremos, para comprobar sus experiencias sobre el curso de PSP que han realizado. Las respuestas textuales han sido analizadas mediante el software de análisis cualitativo WebQDA para categorizarlas, poder organizar dichas respuestas y así, realizar las mejoras oportunas para futuros cursos que se realizarán sobre esta misma disciplina. El resultado final del estudio y análisis cualitativo ha permitido mejorar algunas cuestiones concretas que se realizaban de determinada manera en el curso y que en el futuro permitirá cambiar alguna estrategia docente sobre el mismo.
\end{abstract}

Palabras clave: Análisis cualitativo; Ingeniería de Software; Proceso de Software Personal; Cuestionario; webQDA.

\section{QUALITATIVE ANALYSIS OF STUDENT RESPONSES TO IMPROVE THE PERSONAL SOFTWARE PROCESS COURSE}

Abstract. In this article the mixed analysis of the answers given by the students on the teaching of a course carried out in the Software Engineering course and entitled the Personal Software Process (PSP) is carried out. These responses, for later analysis, have been divided into four groups, attending to each of the questions asked, and for each of them the analysis categories have been obtained. These categories of analysis have allowed us to group the different responses that the students have given to a questionnaire that we will later show, to check their experiences on the PSP course they have taken. The textual responses have been analyzed using the WebQDA qualitative analysis software to categorize them, to organize these responses and thus, make the appropriate improvements for future courses to be carried out on this same discipline. The final result of the qualitative study and analysis has made it possible to improve some specific questions that were carried out in a certain way in the course and which in the future will allow us to change some teaching strategy about it.

Keywords: Qualitative analysis; Software Engineering; Personal Software Process; Questionnaire; webQDA.

\section{INTRODUCCIÓN}

El trabajo de un Ingeniero que construye software (Pressman, 2010) debería permitir entregar el mismo con una alta calidad, en el plazo de tiempo estipulado inicialmente y dentro de los costes que se hayan establecido a priori. Esto no siempre es posible y los 
motivos pueden ser de ámbitos variados: desde una mala planificación inicial, pasando por la inexperiencia de los implementadores, hasta un proceso de construcción de software mal realizado. Por todos estos motivos, y otros adicionales, surge el Proceso de Software Personal (en adelante PSP) (Humphrey, 2001), dedicado a enseñar a los Ingenieros Informáticos los principios de la construcción de software desde el punto de vista personal y de la calidad del producto.

EI PSP proporciona una experiencia real a los Ingenieros de Software y les permite realizar el trabajo de construcción integrándolos en un proyecto donde se pasa por las diferentes fases y por las diferentes tareas que hay que realizar en cada una de ellas, incluyendo las fases siguientes: planificación inicial, el diseño, la codificación, la revisión del código, la compilación y las pruebas; todas ellas antes de realizar la entrega del producto al cliente. Pero, el PSP, no solo enseña las tareas que hay que realizar en estas fases, sino que además enseña a los estudiantes a aprender a tomar medidas, es decir, a averiguar, por ejemplo, los tiempos que invierten en realizar las tareas y también cómo aprender de los defectos que cometen, de tal forma que les permitan mejorar con el tiempo y generar software con más calidad en los proyectos futuros. Para ello, los alumnos deben ir adquiriendo sus experiencias y sus conocimientos de su forma de trabajar con las prácticas, es por esto, por lo que en el proceso de enseñanza del PSP se realizan diversas experiencias prácticas para que los alumnos conozcan el tiempo que invierten en realizar los productos y los fallos que comenten al realizarlos. Aprender de ellos, para que les permita mejorar sus ratios invertidos en los próximos proyectos que realicen.

EI PSP es un proceso personal que debe realizar cada estudiante y que consiste en tomar y almacenar los datos reales de los trabajos que realiza el propio ingeniero para estimar el tamaño, el esfuerzo (tiempo) y la calidad (en cuanto a defectos introducidos) de los nuevos trabajos futuros. Este proceso se realiza aproximadamente en 15 horas lectivas y para ello los estudiantes trabajan 12 Kits o prácticas en las que van desarrollando todo el PSP y lo van aprendiendo mientras las realizan.

\section{OBJETIVOS}

Llevamos impartiendo el curso presencial de PSP durante varios años, y nos interesaba conocer la opinión, de los propios alumnos involucrados en el curso, de cómo se estaba llevando a cabo el mismo, de tal forma que nos permitiera comprobar si realmente este curso les servía de ayuda en su formación como Ingenieros y de qué forma. Nos 
preguntamos, inicialmente, por algunas cuestiones que teníamos la incertidumbre de si se estaban o no produciendo, entre las que destacamos:

1) ¿Cuál es la percepción de los estudiantes en cuanto a si esta formación les ayudará realmente o no como Ingeniero?

2) ¿Cuáles serán los aspectos positivos que más valorarán los estudiantes?

3) ¿Cuáles serán los aspectos más negativos de este proceso para los estudiantes?

Ante esta incertidumbre propusimos tres objetivos en nuestra investigación para intentar dar respuesta a cada una de las preguntas anteriormente formuladas. A saber:

1) Conocer la opinión de los alumnos sobre si este curso de PSP les ayuda en la mejora personal como Ingeniero Informático.

2) Obtener, desde el punto de vista del estudiante, cuáles son los aspectos más positivos que tiene este proceso.

3) Obtener, desde el punto de vista del estudiante, cuáles son los aspectos más negativos que tiene este proceso.

Las preguntas de investigación se las trasladamos a los alumnos para que, de forma abierta, dieran sus opiniones en un cuestionario y respondieran a las mismas. Es por ello, por lo que fue necesario analizar sus respuestas textuales mediante algún software de análisis cualitativo, en este caso elegimos WebQDA (Costa, Linhares \& De Souza, 2012) como herramienta de análisis.

\section{METODOLOGÍA}

En esta investigación se hace uso de metodologías mixtas (Hernández, Fernández \& Baptista, 2014), tanto cuantitativas como cualitativas, para analizar las respuestas de los alumnos. Se ha utilizado el software de análisis estadístico SPSS (Molina-Fernández, 2010) para analizar de forma cuantitativa el resultado de las respuestas de los alumnos a la primera pregunta. Se ha usado el software de análisis cualitativo WebQDA (Souza, Costa \& Moreira, 2011) para analizar de forma cualitativa el resultado de las respuestas textuales de los alumnos a la segunda y tercera pregunta. Para estas dos últimas preguntas, se introdujeron como fuentes internas las respuestas de los alumnos, se analizaron los contenidos textuales de los mismos realizando un análisis cualitativo y se obtuvieron las categorías encontradas en las respuestas, lo que ha permitido categorizar las respuestas y 
obtener la información que buscamos. Las categorías de análisis han emergido desde los datos en función de las ideas que mencionan los participantes y se han buscado las ideas o patrones similares para realizar la organización de las mismas. Estudios similares al que se ha llevado a cabo en esta investigación, lo han realizado otros investigadores, aunque en otros entornos diferentes como: en el aseguramiento de la calidad en el proceso de desarrollo de software (Chavarria, Ore \& Pastor, 2016; Delgadillo, Abud-Figueroa, PeláezCamarena, Alor-Hernández \& García, 2016) y en experiencias académicas (Gómez, Aguileta, Gómez \& Aguilar, 2017).

Existen otros artículos donde se han realizado experiencias similares a la que hemos hecho nosotros con clases de alumnos, entre ellas podemos destacar las realizadas en (Abrahamsson \& Kautz, 2002; Carrington, McEniery \& Johnston, 2001). En ellas también se han trabajado con estudiantes universitarios de licenciatura o Grado, en cursos similares como $3^{\circ} \circ 4^{\circ}$, con un número de estudiantes parecido a nuestra muestra y utilizando como lenguajes de programación Java para realizar los proyectos. Posteriormente, compararemos los resultados obtenidos en estos trabajos con los resultados que hemos obtenidos nosotros.

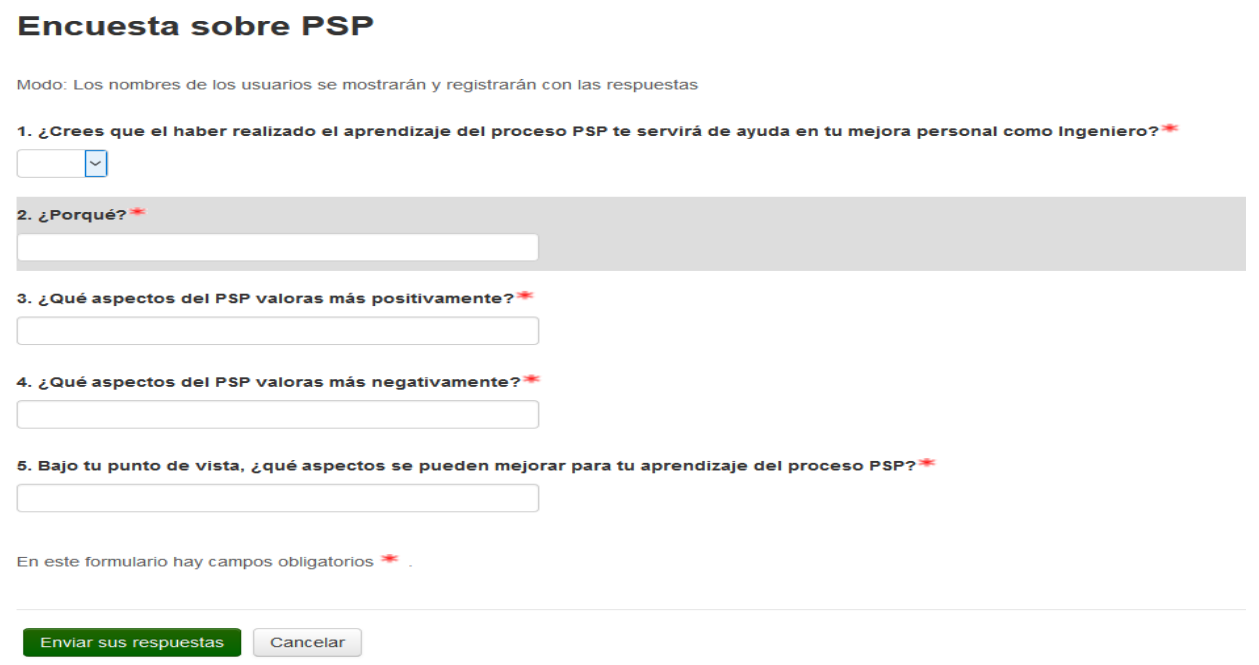

Figura 1. Cuestionario sobre el Proceso de Software Personal (PSP).

La Figura 1 muestra el cuestionario (Arias, 2008) que se ha elaborado y que va a ser utilizado para realizar la encuesta a los alumnos. El cuestionario se ha implementado dentro del curso virtual de Moodle en la asignatura de Ingeniería de Software, de $3^{\text {er }}$ curso, en el Grado en Ingeniería Informática en Tecnologías de la Información que se imparte en el 
Centro Universitario de Mérida de la Universidad de Extremadura (España). Esta asignatura actualmente se imparte en el primer semestre del curso (de Septiembre a Febrero). El cuestionario se realizó al finalizar el curso de PSP ya que en ese momento los alumnos tenían una visión completa y reciente del todo el trabajo realizado durante el curso y una estimación veraz del propio PSP.

El cuestionario tiene cinco preguntas diferenciadas, de las cuales para nuestra investigación solo utilizaremos las preguntas 1, 3 y 4 del mismo. La primera pregunta la responderán los alumnos con una respuesta de tipo $\mathrm{S}$ o $\mathrm{N}$, indicando si este aprendizaje les sirve de ayuda (S) en su formación como Ingeniero Informático, o en caso contrario no $(\mathrm{N})$. La tercera y cuarta pregunta son preguntas abiertas en las que el alumno puede introducir un texto; la pregunta 3 utilizada para aportar los aspectos positivos que perciben del PSP para su formación, y la pregunta 4 para aportar los aspectos negativos.

Las preguntas del cuestionario son las siguientes:

- Ítem 1: ¿Crees que el haber realizado el aprendizaje del PSP te servirá de ayuda en tu mejora personal como Ingeniero? Respuestas posibles $(\mathrm{S} / \mathrm{N})$.

- Ítem 2: ¿Por qué? Respuesta abierta (textual).

- Ítem 3: ¿Qué aspectos del PSP valoras más positivamente? Respuesta abierta (textual).

- Ítem 4: ¿Qué aspectos del PSP valoras más negativamente? Respuesta abierta (textual).

- Ítem 5: Bajo tu punto de vista, ¿qué aspectos se pueden mejorar para tu aprendizaje del proceso PSP? Respuesta abierta (textual).

Hemos usado el análisis cualitativo, al que nos hemos referido anteriormente, para analizar las respuestas dadas por los alumnos a las preguntas de los ítems 3 y 4 . En el cuestionario mostrado en la Figura 1 los ítems 2 y 5 no son utilizados en esta investigación.

Se eligió una muestra no probabilística por cuotas de alumnos (Martín-Marín, RamosSánchez \& Cubo-Delgado, 2011), para que supiéramos con seguridad que han cursado la asignatura antes indicada, en este caso concreto durante los últimos tres cursos.

\section{RESULTADOS Y DISCUSIÓN}


Los 35 alumnos que colaboraron en la encuesta realizaron y enviaron sus respuestas, de cada ítem del cuestionario (Alaminos \& Castejón, 2006), si lo creyeron oportuno, ya que no todos los alumnos realizaron sugerencias textuales, a través del cuestionario realizado en Moodle, a todos los ítems del mismo. Con toda la información recogida se generaron 5 ficheros, uno por cada ítem, con las respuestas textuales dadas por ellos. Podemos ver un ejemplo en la Figura 2 que corresponde al fichero que contiene alguna de las respuestas realizadas por los alumnos al ítem 3 del cuestionario, es decir, el ítem que contempla los aspectos positivos que valoran los alumnos una vez realizado el PSP.

\begin{tabular}{|c|c|}
\hline Alumno & ¿Qué aspectos del PSP valoras más positivamente? \\
\hline alu1 & El modelo de medición de errores dentro de los proyectos software. \\
\hline alu2 & La organización de cara a realizar tareas. \\
\hline alu3 & Que me haga hacer la planificación y calcular lo que tardo. \\
\hline alu4 4 & La ayuda que te ofrece a la hora de saber dónde estás perdiendo el tiempo. \\
\hline alus & $\begin{array}{l}\text { aprender a realizar las diferentes fases (planificación, diseño, codificación, compilación y } \\
\text { pruebas) correctamente }\end{array}$ \\
\hline alu6 & $\begin{array}{l}\text { Valoro la capacidad de organización y perfeccionamiento del tiempo personal, para así ser más } \\
\text { provechoso }\end{array}$ \\
\hline alu7 & intentar tardar menos tiempo y no perderlo \\
\hline alu8 & $\begin{array}{l}\text { El uso de las tablas es intuitivo. Ves el que se consume tu tiempo, no solo en el nivel de Ing. de } \\
\text { software }\end{array}$ \\
\hline alu9 & La organización a la hora de hacer un proyecto \\
\hline alu10 & $\begin{array}{l}\text { Las tablas sobre el control del código que hemos realizado, ya que creo que pueden acabar } \\
\text { ayudándonos en futuros desarrollos y a mejorar la manera en la que programamos }\end{array}$ \\
\hline alu11 & La capacidad de prevenir \\
\hline alu12 & Ayudarme a analizar más en profundidad problemas. \\
\hline alu13 & $\begin{array}{l}\text { El ayudarnos a detectar la cantidad de defectos que cometemos programando y que solemos } \\
\text { pasar por alto. }\end{array}$ \\
\hline alu14 & $\begin{array}{l}\text { Lo que más valoro de PSP es todo el proceso que hay que hacer antes de hacer un programa, si } \\
\text { se hace una buena planificación, el resultado será positivo en menor tiempo. }\end{array}$ \\
\hline alu15 & $\begin{array}{l}\text { Valoro su propósito final para ayudar a un desarrollo software eficiente y cometiendo cada vez } \\
\text { menos errores, basándonos en el estudio de experiencias previas. }\end{array}$ \\
\hline alu16 & Que hace registro del tiempo empleado \\
\hline alu17 & El análisis de los errores cometidos para aprender de ellos \\
\hline alu18 & El resultado que te proporciona al final del proceso \\
\hline alu19 & La organización, la mejora de visión a la hora de mejorar ciertas fases \\
\hline alu20 & El saber en que fase cometo los errores. \\
\hline alu21 & $\begin{array}{l}\text { El aspecto que más valoro es que he aprendido una técnica para poder aumentar mi } \\
\text { rendimiento en el desarrollo de mi software en cuanto a lo que errores se refiere. }\end{array}$ \\
\hline alu22 & $\begin{array}{l}\text { Poder obtener un software de calidad, a partir de los procesos realizados en el PSP. Es decir, } \\
\text { dedicar más tiempo en la revisión del código, realizar un diseño antes de implementar el código, } \\
\text { etc. }\end{array}$ \\
\hline alu23 & La organización semanal. Saber a la semana cuanto tiempo has invertido en diferentes tareas. \\
\hline alu24 & La corrección de errores \\
\hline alu25 & que te ayuda a organizarte mejor \\
\hline alu26 & me ayuda a administrar mi tiempo y a ser más productivo y organizado \\
\hline alu27 & Las pruebas que realizamos en el mismo \\
\hline
\end{tabular}

Figura 2. Sugerencias realizadas por los alumnos al ítem 3.

Teniendo en cuenta que la muestra proporcionada es pequeña (35 alumnos), se realizaron pruebas de normalidad, observando, en la Tabla 1, que no existe normalidad en la muestra, ya que el valor de Sig. es 0 , tanto en la prueba de Kolmogorov-Smirnov como en la prueba e Shapiro-Wilk. Es por ello, por lo que se utilizarán pruebas No paramétricas en las siguientes pruebas que se realicen. 
Tabla 1. Pruebas de Normalidad.

\begin{tabular}{|c|c|c|c|c|c|c|}
\hline & \multicolumn{3}{|c|}{$\begin{array}{c}\text { Kolmogorov- } \\
\text { Smirnov }\end{array}$} & \multicolumn{3}{c|}{ Shapiro-Wilk } \\
\cline { 2 - 7 } & $\begin{array}{c}\text { Estadísti } \\
\text { co }\end{array}$ & gl & Sig. & $\begin{array}{c}\text { Estadísti } \\
\text { co }\end{array}$ & gl & Sig. \\
\hline $\begin{array}{c}\text { ¿Has mejorado con el proceso } \\
\text { PSP? }\end{array}$ &, 533 & 35 &, 000 &, 317 & 35 &, 000 \\
\hline
\end{tabular}

a. Corrección de significación de Lilliefors

Para dar respuesta a la pregunta: ¿Crees que el haber realizado el aprendizaje del PSP te servirá de ayuda en tu mejora personal como Ingeniero? Se realizará la prueba No paramétrica binomial exacta. Los resultados obtenidos de forma descriptiva se pueden observar en la Tabla 2. Los resultados de significación de la prueba binomial exacta se pueden observar en la Tabla 3.

En esta última se observa que el valor la significación exacta (bilateral) es 0 , lo que implica que la mayoría de los alumnos han respondido afirmativamente a esta pregunta, es decir, los alumnos opinan que el aprendizaje del curso de PSP les ha ayudado en su mejora personal como ingeniero informático.

Tabla 2. Estadísticos descriptivos.

\begin{tabular}{|c|r|r|r|r|r|}
\hline & $\mathbf{N}$ & Media & $\begin{array}{c}\text { Desviación } \\
\text { estándar }\end{array}$ & Mínimo & Máximo \\
\hline $\begin{array}{c}\text { ¿Has mejorado con el proceso } \\
\text { PSP? }\end{array}$ & 35 &, 086 &, 2840 &, 0 & 1,0 \\
\hline
\end{tabular}

Tabla 3. Prueba estadística binomial exacta.

\begin{tabular}{|c|r|r|r|r|r|r|}
\hline \multicolumn{2}{|c|}{} & Categoría & N & $\begin{array}{c}\text { Prop. } \\
\text { observad } \\
\text { a }\end{array}$ & $\begin{array}{c}\text { Prop. } \\
\text { de } \\
\text { prueba }\end{array}$ & $\begin{array}{c}\text { Significación } \\
\text { exacta } \\
\text { (bilateral) }\end{array}$ \\
\hline $\begin{array}{c}\text { ¿Has mejorado con el proceso } \\
\text { PSP? }\end{array}$ & $\begin{array}{l}\text { Grupo 1 } \\
\text { Grupo 2 }\end{array}$ &, 0 & 32 &, 91 &, 50 &, 000 \\
\hline & Total & & 35 & 1,09 & & \\
\hline
\end{tabular}

Como resultado del análisis cualitativo realizado, a todas las sugerencias de los alumnos para los ítem 3 (aspectos positivos del PSP) y 4 (aspectos negativos del PSP), se obtuvieron las siguientes categorías junto con el número de veces que han aparecido cada una de ellas, tal como se puede observar en las siguientes Tabla 4 y Tabla 5. 
Tabla 4. Nombres de las categorías de aspectos positivos con el número de veces que aparecen.

\begin{tabular}{lc}
\hline \multicolumn{1}{c}{ Nombre categoría } & $\begin{array}{c}\text { Núm. } \\
\text { referencias }\end{array}$ \\
\hline Aspectos positivos del proceso PSP & \\
Mejorar la planificación de los & 3 \\
proyectos & 11 \\
Optimizar las tareas & 7 \\
Conocer y prever los defectos & 13 \\
Conocer, estimar y aprovechar el & 12 \\
tiempo & \\
Mejorar la calidad del proceso & \\
\hline
\end{tabular}

La Tabla 4 se corresponde con la distribución de las categorías obtenida en el análisis cualitativo para los aspectos positivos del PSP. Se han obtenido cinco categorías que procedemos a comentar a continuación:

- La primera categoría se refiere a que la realización de este proceso personal les ha supuesto a los alumnos una mejora para realizar la planificación de nuevos proyectos de software, en concreto nos hemos referido a ella como: "Mejorar la planificación de los proyectos". El número de referencias obtenido para esta categoría han sido solo tres, y se corresponde a la que menor número de referencias ha obtenido por parte de los alumnos. Aunque este aspecto es uno de los que más énfasis hace el PSP, sin embargo, para los alumnos es al que menos importancia le dan. De hecho, las prácticas de programación se hacen comenzando con la planificación de un nuevo proyecto y se continúa con las anotaciones de los datos reales del mismo.

- La segunda categoría se refiere a que la realización de este proceso ha supuesto para los alumnos la optimización y mejora de las tareas que realizan en cada una de las fases del proceso de construcción de software, en concreto nos referimos a ella como: "Optimizar las tareas". Se encuentra entre las tres categorías más referidas por los alumnos con once referencias. Está claro que los alumnos aprecian significativamente la realización práctica de proyectos informáticos, donde les quedan claramente establecido las taras que deben realizar antes, durante y después del desarrollo del mismo, aunque esto no sea lo fundamental del PSP.

- La tercera categoría se refiere al conocimiento y la previsión de los fallos que cometen los Ingenieros de Software, en este caso los alumnos, durante el proceso de construcción del mismo. En concreto nos referimos a ella como: "Conocer y 
prever los defectos". En este caso, a esta categoría le dan una importancia relativa mínima ya que es la segunda menos referenciada con siete referencias. Es curioso, porque el PSP precisamente hace mucho énfasis en este aspecto como una de las formas de obtener la calidad en la construcción de software, ya que la misma viene a través de la minimización en la introducción de defectos en la construcción; incluso introduce la fase de revisión de código precisamente las detectar y corregir los defectos antes de la compilación y pruebas del software. Sin embargo, los alumnos no aprecian suficientemente este aspecto con la importancia que tiene.

- La cuarta categoría sirve para aglutinar en ella aquellas opiniones emitidas por los alumnos con respecto a lo positivo que les ha sido el conocer cómo invierten su tiempo en el proceso, cómo aprenden a estimar el tiempo que van a tardar en construir nuevo software y dónde invierten el tiempo en la construcción. En concreto nos referimos a ella como: "Conocer, estimar y aprovechar el tiempo". En efecto, esta es la categoría que más positivamente han estimado los alumnos con trece referencias. En esto coinciden con el PSP, es decir, una de las cuestiones más importantes que enseña el proceso es aprender a conocer cuánto tiempo invierten en la construcción de software y cada una de las fases de construcción, además, de aprender a estimar el tiempo que van a invertir en la construcción de nuevo software. Parece que los alumnos estiman muy positivamente este aspecto y coinciden con una de las cuestiones más importante del PSP.

- La quinta categoría se refiere a las opiniones que han realizado los alumnos en general con respecto a todo el proceso de construcción de software aprendido. En concreto, nos referimos a ella como: "Mejorar la calidad del proceso". Ellos aprecian muy positivamente la mejora de su proceso de construcción de software, siendo esta categoría la segunda más referenciada con doce referencias. En esto coinciden con lo que quiere enseñar el PSP, es decir, el PSP intenta enseñar un proceso de construcción de software con la calidad suficiente para que el mismo tenga el menor número de defectos $\mathrm{y}$, parece que al realizar el curso de PSP nuestros alumnos también aprecian que su proceso de construcción personal de software ha mejorado de tal forma que lo realizan con la calidad suficiente conforme a las directrices aprendidas en el curso. 
INVESTIGAÇÃO QUALITATIVA EM CIÊNCIAS SOCIAIS: AVANÇOS E DESAFIOS | INVESTIGACIÓN CUALITATIVA EN CIENCIAS SOCIALES: AVANCES Y DESAFIOS

Tabla 5. Nombres de las categorías de aspectos negativos con el número de veces que aparecen.

\begin{tabular}{lc}
\hline \multicolumn{1}{c}{ Nombre categoría } & $\begin{array}{c}\text { Núm. } \\
\text { referencias }\end{array}$ \\
\hline Aspectos negativos del proceso PSP & \\
El registro del tiempo u otra información & 12 \\
Estimar los defectos & 3 \\
No se cree en el proceso PSP & 9 \\
Dificultad de aplicar el proceso PSP & 9 \\
La utilidad y rigidez de algunas tablas & 5 \\
Nada es negativo & 1 \\
\hline
\end{tabular}

La Tabla 5 corresponde a la distribución de las categorías obtenida en el análisis cualitativo para los aspectos negativos del PSP. Se han obtenido seis categorías que procedemos a comentar a continuación:

- La primera categoría se refiere a la toma de datos, en cuanto al registro del tiempo y de otra información, en las tablas utilizadas en este proceso personal. En concreto nos referimos a ella como: "El registro del tiempo u otra información". El número de referencias obtenido para esta categoría han sido doce, que se corresponde a la que mayor número de referencias ha obtenido por parte de los alumnos. Sin lugar a dudas, lo que menos les gusta a los alumnos es registrar la información. No obstante, entendemos que pueden llevar su parte de razón ya que se pudiera automatizar mejor todo este proceso de toma de datos. Esta puede ser una cuestión a tener en cuenta como mejora en el futuro.

- La segunda categoría se refiere a forma que tiene este proceso de recoger la información para detectar y mejorar lo referente a los defectos. En concreto nos referimos a ella como: "Estimar los defectos". Se encuentra entre las dos categorías que consideran, los alumnos, como menos negativo, con solo tres referencias. Esto iría en contra de una de las cuestiones que precisamente persigue este PSP, es decir, aprender a saber dónde y cómo cometemos errores para mejorar. Una cuestión interesante para incidir en ella en los próximos cursos.

- La tercera y la cuarta categoría se refieren a que el alumno, pese a llevar a cabo el proceso de PSP, no ve que le sirve de ayuda en su trabajo y, a que cuando lo realizan detectan alguna dificultad, respectivamente. En concreto nos referimos a ellas como: "No se cree en el proceso PSP" y "Dificultad de aplicar el proceso PSP". En este caso, a estas dos categorías les dan una importancia relativa media-alta, ya que es la segunda más referenciada con nueve referencias. Considero que los 
alumnos, cuando cursan esta asignatura, vienen con unas marcadas pautas de programación sin ninguna metodología concreta, por lo que reconfigurar sus malos hábitos adquiridos es complicado. Todo lo que esté fuera de lo estrictamente necesario para programar no lo tienen en cuenta suficientemente como que les es beneficioso. Creemos que están equivocados y precisamente queremos hacerles ver con este proceso sus creencias. Quizás sea necesario más tiempo para conseguirlo.

- La quinta categoría sirve para aglutinar en ella aquellas opiniones emitidas por los alumnos con respecto a lo negativo que le parece el hacer uso de determinadas tablas e incluso del contenido que tienen las mismas. En concreto nos referimos a ella como: "La utilidad y rigidez de algunas tablas". Esta categoría la han estimado los alumnos con cinco referencias. Aunque el proceso es repetitivo con la anotación en tablas de los tiempos en los que realizan sus tareas para coger hábitos, es posible que, en algunos casos, y alguna tabla muy concreta se pudiera mejorar para que se centraran en las necesidades estrictamente necesarias.

- La sexta categoría se refiere a no hay nada negativo en este proceso. En concreto nos referimos a ella como: "Nada es negativo". Algún alumno, ya que solo hay una referencia, cree que todo está correcto en el proceso.

\section{CONCLUSIONES}

Como conclusiones obtenidas después de realizar la investigación mixta, siendo todo lo cauto necesario en este tipo de investigación y teniendo en cuenta el tamaño muestral, hemos de indicar las siguientes cuestiones:

Estadísticamente de modo significativo, los alumnos consideran que la realización del curso que implementa el aprendizaje del Proceso de Software Personal (PSP) es positivo como enseñanza para su futuro como Ingeniero Informático. Esto es interesante teniendo en cuenta el trabajo invertido en la preparación y realización de este tipo de cursos, ya que su apreciación permite continuar organizando cursos, como este o similares, en el futuro.

A las sugerencias que nos aportan los alumnos consultados, tanto en los aspectos positivos como negativos, en algunos casos coinciden con estudios anteriores como los aportados por (Abrahamsson \& Kautz, 2002; Carrington et al., 2001; Gómez et al., 2017), sin embargo, también introducen algún aspecto novedoso que estos estudios no contemplan. 
Como coincidencias de nuestra investigación con respecto a los estudios anteriores tenemos que: el PSP permite mejorar las estimaciones de los alumnos en cuanto al tamaño del producto y al esfuerzo que van a realizar, también les permite mejorar la calidad con la que realizan la construcción de nuevo software, coinciden también en las quejas sobre el esfuerzo y tiempo requerido para recolectar la información y lo dificultoso y tedioso que es este proceso y, por último, coinciden también en la falta de motivación o falta de disciplina en la realización del proceso PSP, ya que este, les hace modificar su forma de trabajar habitual, como por ejemplo: tener que hacer las revisiones antes de la compilación o tener que terminar la codificación antes de realizar la depuración.

Como cuestiones novedosas que no son recogidas o comprobadas en los estudios citados anteriormente, nuestros alumnos coinciden ampliamente en que este proceso les ha permitido ser más productivos en la construcción de software ya que les ha permitido optimizar la realización de algunas de sus tareas habituales.

También aportan la necesidad de mejorar la utilización de algunas tablas, al menos el uso tan reiterativo que se hace de ellas. Por ejemplo, la realización de las tablas de Registro de tiempos y Registros semanal de actividades, tienen una función necesaria que es el habituar al alumno en la toma de tiempos, pero que se hacen menos necesarias cuando entra en juego el Plan de proyecto, ya que es este documento el que recogerá la información fundamental e imprescindible de todos los aspectos del proyecto.

Este último aspecto, permitirá mejorar del proceso de enseñanza-aprendizaje en aquellas cuestiones que realmente estimamos pertinentes a realizar en próximos cursos.

\section{Agradecimentos}

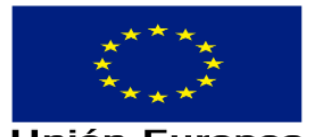

Unión Europea

Fondo Europeo de Desarrollo Regional. Una manera de hacer Europa.

\section{REFERENCIAS}

Abrahamsson, P. \& Kautz, K. (2002). Personal software process: Classroom experiences from Finland. In European conference on Software Quality (pp. 175-185). Springer, Berlin, Heidelberg.

Alaminos, A. \& Castejón, J. L. (2006). Elaboración, análisis e interpretación de encuestas, cuestionarios y escalas de opinión. Alicante. Universidad de Alicante. 
Arias, J. (2008). Evaluación de la Calidad de Cursos Virtuales: Indicadores de Calidad y construcción de un cuestionario de medida. Aplicación al ámbito de asignaturas de Ingeniería Telemática. Memoria para el título de Doctor. Badajoz: Universidad de Extremadura.

Carrington, D., McEniery, B. \& Johnston, D. (2001). PSP (sm) in the Large Class. Proceeding in 14th Conf. In Software Engineering Education and Training (págs. 81-88). Charlotte, North Carolina (EEUU): IEEE Computer Society.

Chavarria, A.E., Ore, S.B. \& Pastor, C. (2016). Aseguramiento de la calidad en el proceso de desarrollo de software utilizando CMMI, TSP y PSP. RISTI [Revista Iberica de Sistemas e Tecnologias de Información], (20), 62-77.

Costa, A., Linhares, R. \& De Souza, F. (2012). Possibilidades de Análise Qualitativa no webQDA e colaboração entre pesquisadores em educação em comunicação. Conferencia: Infoinclusão e as possibilidades de ensinar e aprender (pág. 276-286). Aracaju: ANAIS.

Delgadillo, M.L.U., Abud-Figueroa, M.A., Peláez-Camarena, S.G., Alor-Hernández, G. \& García, A. I. S. (2016) Propuesta de un modelo de integración de PSP y Scrum para mejorar la calidad del proceso de desarrollo en una MiPyME. Research in Computing Science, 120, 147-157.

Gómez, O.S., Aguileta, A.A., Gómez, G.E. \& Aguilar, R. A. (2017). Estudio del Proceso Software Personal (PSP) en un entorno académico. ReCIBE, Revista electrónica de Computación, Informática, Biomédica y Electrónica, 3(2), 1-28.

Hernández, R., Fernández, C. \& Baptista, P. (2014). Metodología de la investigación. México: McGraw-Hill.

Humphrey, W.S. (2001). Introducción al Proceso Software Personal. Madrid: Pearson Educación (Addison Wesley).

Martín-Marín, B., Ramos-Sánchez, J.L. \& Cubo-Delgado, S. (2011). Métodos de Investigación y análisis de datos en ciencias sociales y de la salud. Piramide.

Molina-Fernández, M. (2010). Introducción a la inferencia estádística. Cáceres, Cáceres, España: Universidad de Extremadura.

Pressman, R.S. (2010). Ingeniería de Software: un enfoque práctico (7ª Edicion). Mexico: McGraw-Hill.

Souza, F.N., Costa, A.P.\& Moreira, A. (2011). Análise de Dados Qualitativos Suportada pelo Software WebQDA. VII Conferência Internacional de TIC na Educação: Perspetivas de Inovação, (págs. 49-56). Braga (Portugal). 